\title{
Urogenital Involvement in the Klippel-Trenaunay-Weber Syndrome. Treatment Options and Results
}

\author{
Fabio C. Vicentini, Francisco T. Denes, Cristiano M. Gomes, Alexandre Danilovic, Frederico \\ A. Silva, Miguel Srougi
}

Division of Urology, University of Sao Paulo School of Medicine (USP), Sao Paulo, Brazil

\begin{abstract}
Objective: Klippel-Trenaunay-Weber syndrome (KTWS) is a congenital condition characterized by vascular malformations of the capillary, venous and lymphatic systems associated to soft tissue and bone hypertrophy in the affected areas. This syndrome may involve bladder, kidney, urethra, ureter and genitals. We report the treatment of 7 KTWS patients with urogenital involvement.

Materials and Methods: From 1995 to 2005, 7 patients with KTWS were evaluated and the charts of these patients were reviewed.

Results: Patients' median age was 19-years (range 4 to 46-years) and only 1 was female. The clinical presentation included genital deformities in 3 cases, hematuria in 2 and urethrorragia in 2, one of which associated with cryptorchidism and phimosis. Three patients had an association of pelvic and genital malformations, including 2 patients with hematuria due to vesical lesions and 1 patient with left ureterohydronephrosis due to a pelvic mass. Two patients had urethral lesions. Treatment included endoscopic laser coagulation for 1 patient with recurrent hematuria and 1 patient with urethrorrhagia, pelvic radiotherapy for 1 patient with hematuria and circumcision in 2 patients with genital deformities. One patient required placement of a double-J catheter to relieve obstruction. Hematuria and urethrorragia were safely and effectively controlled with laser applications. Circumcision was also effective. The patient treated with radiotherapy developed a contracted bladder and required a continent urinary diversion.

Conclusions: Urogenital involvement in patients with KTWS is not rare and must be suspected in the presence of hematuria or significant cutaneous deformity of the external genitalia. Surgical treatment may be warranted in selected cases.
\end{abstract}

Key words: Klippel-Trenaunay-Weber syndrome; urogenital system; hematuria, lasers, bladder

Int Braz J Urol. 2006; 32: 697-704

\section{INTRODUCTION}

Klippel-Trenaunay-Weber Syndrome (KTWS) is a rare congenital syndrome characterized by vascular malformations of the capillary, venous and lymphatic systems associated to soft tissue and bone hypertrophy of an affected lower limb. These alterations are frequently misdiagnosed as simple hemangiomas, which have a different clinical behavior (1).

Historically, Klippel and Trenaunay described the first case of KTWS in 1900 and in 1907 Weber described similar cases. They believed that the syndrome was secondary to large congenital arteriovenous fistulae, causing hypertrophy of the affected limb (2). Recently, Tian et al. identified an 
angiogenic factor, termed VG5Q, that when over expressed due to mutations on its gene, causes increased angiogenesis. This is accepted as the molecular pathogenic mechanism of KTWS (3).

There is considerable variability in the terminology for these similar syndromes. The diagnosis of the Klippel-Trenaunay Syndrome should be applied to cases without arteriovenous fistulae, while Weber or Parks-Weber Syndrome is more appropriate for cases with arteriovenous fistulae (4). Since the differentiation between the two conditions is only possible by means of histopathological, radiological and/or genetic evaluation, we employ the name Klippel-Trenaunay-Weber Syndrome, as recommended by other authors (5).

Involvement of genitourinary organs is not rare in patients with KTWS. The bladder is estimated to be affected in $2.3 \%$ to $6 \%$ of the cases (1). Hematuria is usually the initial clinical manifestation $(1,2)$. The urethra, external genitalia, kidney and ureter may also be involved (1).

In this study, we report our experience with seven patients with KTWS and urogenital involvement.

\section{MATERIALS AND METHODS}

We retrospectively reviewed the records of seven consecutive patients (6 men and 1 woman) evaluated over a period of 10 years, who were referred to urological evaluation with a previously established diagnosis of KTWS. The criteria employed for the KTWS diagnosis were the typical clinical and physical features.

Urological assessment and management varied according to the patient's complaints and affected organs. A focused history and physical examination was performed in all cases. Laboratorial and imaging studies were obtained according to clinical presentation.

\section{RESULTS}

The bladder and external genitalia were the most commonly affected organs in our series. Symp- toms presented were penile deformity in three patients, recurrent gross hematuria in two, urethral bleeding in one and phimosis and cryptorchidism in one. One patient had both penile deformity and unilateral hydronephrosis, due to entrapment of the bladder and distal segment of the left ureter secondary to a large pelvic malformation. The patients' data, urogenital structures involved and urological management are depicted in Table-1.

Case 1: A 46-year-old man had a history of gross hematuria since childhood. He had a typically enlarged right leg and penile varicosities. He underwent cystoscopy elsewhere at the age of 21 revealing a large hemangiomatous bladder lesion. He was treated with external beam radiotherapy, and developed actinic cystitis that evolved to a contracted bladder with lithiasis. A continent diversion with a sigmoid segment was performed 11 years later, and he remained free of hematuria and other symptoms for the following 14 years.

Case 2: A 4-year-old boy presented an enlarged right leg and a lymphedematous enlarged penis and preputial skin that caused voiding difficulty. He was previously treated elsewhere by inguinal lymphatic-venous diversion and circumcision. Penile enlargement and deformity persisted but the voiding symptoms resolved after the foreskin was partially removed. He was managed with further surgical reduction of the lymphedematous tissue of the penis with significant aesthetical improvement.

Case 3: A 5-year-old boy with sickle cell anemia presented an extremely enlarged right leg, penile deformity due to hemangiomatous varicosities, lymphedema (Figure-1) and a palpable pelvic mass. Abdominal ultrasound revealed left ureterohydronephrosis and a pelvic mass involving the bladder. An abdominal CT scan showed a large bladder lesion extending to the left pelvic wall (Figure-2). Cystoscopy demonstrated an intensely vascularized bladder lesion that infiltrated most of the bladder dome and left wall, including the left ureteral orifice. The penile deformity was managed with surgical removal of the hemangiomatous varicosities and a double $\mathbf{J}$ catheter was placed to drain the left kidney. A laparoscopy was performed simultaneously to evaluate the pelvic mass, which was shown to extensively 
Klippel-Trenaunay-Weber Syndrome

Table 1 - Urogenital involvement in Klippel-Trenaunay-Weber syndrome and the respective treatment.

\begin{tabular}{|c|c|c|c|c|c|c|}
\hline Case & Age & Gender & Race & Involved Organs & Symptoms & Urological Management \\
\hline 1 & 46 & Male & Black & Penis, bladder & Recurrent gross hematuria & $\begin{array}{c}\text { Pelvic RDT and continent } \\
\text { diversion }\end{array}$ \\
\hline 2 & 4 & Male & White & Penis & $\begin{array}{l}\text { Voiding difficulty, penile } \\
\text { deformity }\end{array}$ & Postectomy \\
\hline 3 & 5 & Male & White & $\begin{array}{l}\text { Penis, bladder, } \\
\text { left ureter }\end{array}$ & $\begin{array}{l}\text { Pelvic mass, left ureterohy- } \\
\text { dronephrosis, penile deformity }\end{array}$ & $\begin{array}{l}\text { Double J catheter and re- } \\
\text { moval of penile varicosities }\end{array}$ \\
\hline 4 & 15 & Male & White & Urethra & Urethral bleeding & Holmium laser ablation \\
\hline 5 & 25 & Female & Black & Bladder, vulva & $\begin{array}{l}\text { Recurrent gross hematuria, } \\
\text { vulvar deformity }\end{array}$ & Nd:YAG laser ablation \\
\hline 6 & 5 & Male & White & Penis, urethra & $\begin{array}{c}\text { Phimosis, cryptorchidism and } \\
\text { urethral bleeding }\end{array}$ & Postectomy and orchiopexy \\
\hline 7 & 5 & Male & White & Penis & None & Observation \\
\hline
\end{tabular}

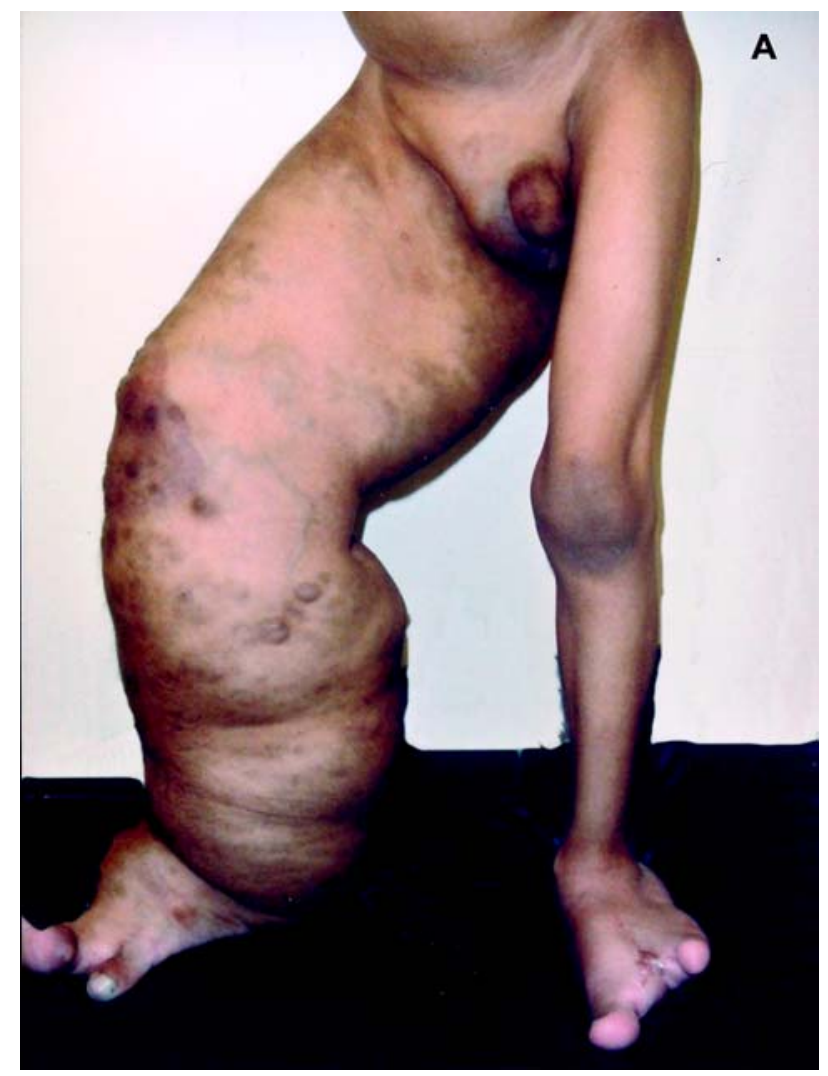

involve the bladder and rectum-sigmoid colon, and was considered irresectable. The patient has been managed conservatively in the last two years with periodic changes of the ureteral catheter, but progressive left renal deterioration occurred due to increasing growth of the pelvic mass. He has also presented recurrent penile varicosities and urethral bleeding that has been managed conservatively.

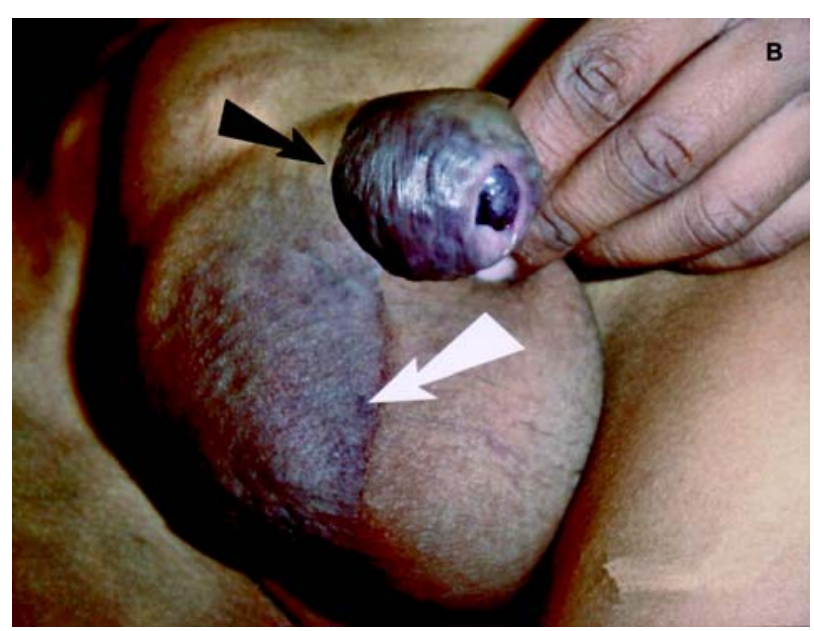

Figure 1 - Five-year-old boy (case \#3) with Klippel-Trenaunay-Weber syndrome. A) Note massive soft tissue hypertrophy and cutaneous vascular malformations of the right leg, perineum and left foot toes. Three left toes amputation were performed. B) Detailed view of penis and scrotum, showing vascular malformation of preputial skin (black arrow) and glans, and right hemiscrotum (white arrow). 


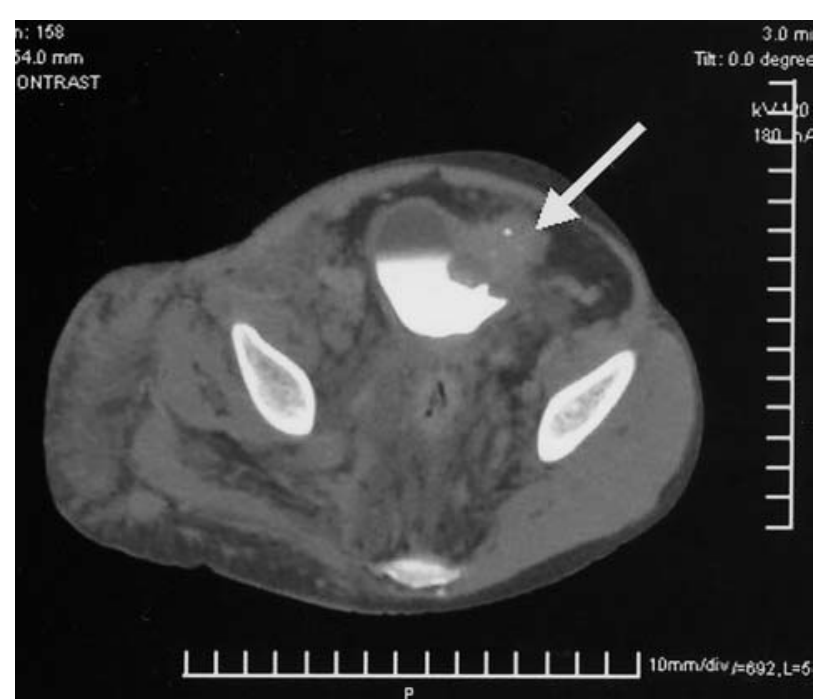

Figure 2 - Pelvic CT scan (case \#3) with intra-vesical contrast medium showing a large lesion involving the left anterior bladder wall (white arrow).

Case 4: A 15-year-old boy presented an enlarged left leg and buttock and a history of intermittent urethral bleeding. Urinary tract sonography was normal. Cystoscopy revealed active bleeding from distal urethral varicose veins, but no bladder lesions. He was treated with Holmium laser application in the urethral varicosities. The laser was used with $6 \mathrm{~W}$ and a $400 \mathrm{~nm}$ fiber. Laser was applied in contact with the urethral mucosa until vascular sclerosis was achieved. A urethral catheter was left for 7 days. After 14 months follow-up, the patient remained free of urethral bleeding.

Case 5: A 25-year-old woman had a diagnosis of KTWS since the age of five. Her medical history included left colectomy at the age of 15 , due to life-threatening enterorrhagia caused by a large pericolonic vascular malformation. She also had a history of sporadic episodes of gross hematuria that were treated conservatively with bladder irrigation, endovenous epsilon amino-caproic acid and occasional blood transfusions. She was referred to our hospital after unsuccessful treatment of recurrent massive hematuria requiring multiple blood transfusions. Physical examination revealed hemangiomas and hypertrophy of the perineum and entire left inferior limb. A suprapubic mass was palpable. Abdomi- nal CT scan revealed diffuse pelvic vascular lesions, involving bladder wall, peri-rectal and paravesical spaces. Cystoscopy revealed a large, elevated and irregular lesion with hemangiomatous features that involved $60 \%$ of the bladder surface on the left, posterior, anterior and dome walls, with well-defined borders and many bleeding sites. The lesion was treated with a continuous pulse Nd:YAG laser, using a 0.6 $\mathrm{mm}$ fiber. The tip of the fiber was positioned $5 \mathrm{~mm}$ from the bladder surface. Total energy delivery was 3,0000 joules in 90 minutes. Bleeding ceased immediately and the bladder catheter was removed on the first postoperative day. After a follow-up period of 14 months, the patient remained free of hematuria.

Case 6: A 5-year-old boy presented enlargement of both hands and left lower limb. He had right cryptorchidism, phimosis and a history of a single episode of urethrorrhagia. The testicle was palpable on the inguinal canal. Many small veins could be seen under the penile skin. Orchiopexy and circumcision were effective, but subcutaneous veins made the dissection of the preputial skin more difficult, requiring ligation of veins and the use of a compressive dressing. At urethrocystoscopy a small vascular malformation was found in the prostatic urethra, with no signs of recent bleeding.

Case 7: A 5-year-old boy was referred for evaluation due to a mild penile and scrotal enlargement associated with a significant enlargement of the left leg. He was otherwise asymptomatic. His pelvic CT scan showed an infiltrating mass in the left buttock, posterior perineum and genital area. The bladder and urethra were not affected and he was managed conservatively.

\section{COMMENTS}

Urogenital involvement in patients with KTWS is not uncommon and may present with different manifestations. Bladder involvement is estimated to occur in $2.3 \%$ to $6 \%$ of the patients, penoescrotal vascular malformations in $8.5 \%$ and vaginal or vulvar in $9.5 \%$ of the patients (1). Cases of kidney, renal artery and ureteral involvement have rarely been reported $(1,6-8)$. Our series is in accor- 
dance with these numbers, with a prevalence of bladder and genital involvement and one patient with ureteral obstruction associated with a massive pelvic vascular malformation.

In cases of extensive cutaneous lesions of the genitals, lower extremities and buttocks, retroperitoneal and urinary tract involvement should be suspected and radiological evaluation with CT scan or MRI is recommended (1). Hematuria and urethral bleeding are also indications for urological evaluation. Bladder and urethral lesions may be confirmed with cystoscopy. In the bladder, they are usually reddish-blue and may be pedunculated, sessile, lobulated or flattened, and are frequently located at the anterior wall or dome (1). Biopsies can lead to massive bleeding and are not recommended.

Initial treatment of gross hematuria in these patients is conservative, with bladder irrigation. Antifibrinolytic agents as epsilon amino-caproic or tranexamic acid can be used (9). In one of our patients (case 5), epsilon was ineffective. Recurrent or life-threatening hematuria demands specific treatment. Partial cystectomy used to be the standard treatment but carries significant morbidity $(10,11)$. Selective embolization of the internal iliac arteries may also be employed, but recurrence due to rapid development of collateral circulation and bladder or prostate infarction has been described (12). Radiotherapy is another therapeutic modality, but only temporary beneficial results are expected, while the morbidity can be excessive (13). In Case 1, radiotherapy resulted in actinic cystitis and loss of a functional bladder, requiring urinary diversion.

The first report of Nd:YAG laser treatment of bladder hemangiomas associated with KTWS was made by Smith and Dixon in 1984 (14). Since then, a few cases have been described, with good results $(12,13,15,16)$. In 1990, Smith reported 13 cases of patients with bladder hemangiomas who underwent treatment with Nd:YAG laser, 6 of whom with KTWS (2). Since then, endoscopic treatment with Nd:YAG laser has been advocated as the gold standard procedure for bladder hemangiomas. In Case 5, the endoscopic approach was used with some technical aspects differing from those described in the literature. We used saline solution to fill the bladder as opposed to $\mathrm{CO}_{2}$ utilized by Kato (12). Since the lesion was very large, our strategy was to initially coagulate the margins of the lesions, in order to decrease blood supply to the bleeding sites. Next, we applied the laser directly to the central areas with large veins, which resulted in bleeding that was easily controlled by applications in the base of the veins. The immediate and mid-term results were excellent, without recurrence of bleeding for more than one year.

The trigone and bladder neck are reported to be rarely involved, but two of our patients (cases 3 and 5) had involvement of the posterior bladder wall and trigone, including obstruction of the left ureteral orifice in Case 3. This is the fourth reported case of KTWS causing hydronephrosis $(1,8)$. Furness et al. described the treatment of one of such cases with ureterolysis. In this case, due to extensive vesical, retroperitoneal and pelvic involvement, the patient was managed conservatively with insertion of a ureteral Double-J catheter to preserve the renal function.

Urethral bleeding is extremely rare in patients with KTWS. We found only one case in the literature, which was treated with excision of the affected urethral segment and full-thickness skin graft. Other cases of urethral bleeding caused by hemangiomas or vascular malformations and not associated to KTWS were treated by excision, Nd:YAG or KTP laser $(17,18)$. Three of our cases had episodes of urethral bleeding. In Case 4, it was controlled with Holmium laser applications on the varicose veins, which to our knowledge represents the first successful report of such treatment. Case 6 had only one self-limited episode of urethral bleeding, and urethrocystoscopy revealed a small prostatic urethra lesion that was not treated. In case 3, the episodes have been mild and sporadic, and no therapy was needed.

Genital lesions are usually managed conservatively. Ulcerations or small bleeding areas are treated with topical antibiotics and compressive dressings. Significant deformity of the penis can be managed with postectomy when necessary. It is usually a safe procedure but may require surgical revision, as occurred in Case 2. Since vascular malformations are not hemangiomas, treatments with steroids, sclero- 
therapy and radiation are not indicated (1). Phimosis can be managed by circumcision. However, careful dissection is necessary, due to the possibility of anomalous enlarged subcutaneous veins, as seen in case 6.

KTWS lesions are not malignant but can have a malignant behavior according to their size and location, as seen in Case 3. Life expectancy for patients with KTWS is not determined in the literature, but adulthood can be reached and many cases of successful pregnancy and delivery have been reported (19). Patients with KTWS must have adequate support and treatment. Vascular and plastic surgeries can ameliorate aesthetical aspects, improving quality of life $(17,20)$. Urologists can have an important role in the care of these patients and must be prepared for that.

\section{CONCLUSIONS}

Urogenital involvement in the KlippelTrenaunay-Weber Syndrome must be suspected when hematuria or urethral bleeding occurs or when extensive cutaneous lesions of the pelvis, genitals, lower extremities and buttocks are present. Imaging studies and urethrocystoscopy confirm the diagnosis. Genital cutaneous malformations may be treated surgically when associated to severe deformity. Hematuria and urethrorrhagia are often managed conservatively, but life-threatening or recurrent episodes should be treated endoscopically. Laser seems to be a good therapeutic option for bleeding vesical or urethral lesions, but the best type of laser for this purpose is yet to be determined.

\section{CONFLICT OF INTEREST}

None declared.

\section{REFERENCES}

1. Furness PD 3rd, Barqawi AZ, Bisignani G, Decter RM: Klippel-Trenaunay syndrome: 2 case reports and a re- view of genitourinary manifestations. J Urol. 2001; 166: $1418-20$.

2. Smith JA Jr: Laser treatment of bladder hemangioma. J Urol. 1990; 143: 282-4.

3. Tian XL, Kadaba R, You SA, Liu M, Timur AA, Yang $\mathrm{L}$, et al.: Identification of an angiogenic factor that when mutated causes susceptibility to Klippel-Trenaunay syndrome. Nature. 2004; 427: 640-5.

4. Ziyeh S, Spreer J, Rossler J, Strecker R, Hochmuth A, Schumacher M, et al.: Parkes Weber or KlippelTrenaunay syndrome? Non-invasive diagnosis with MR projection angiography. Eur Radiol. 2004; 14: 2025-9.

5. Hamsher JB, Farrar T, Moore TD: Congenital vascular tumors and malformations involving urinary tract: diagnosis and surgical management. J Urol. 1958; 80: 299-310.

6. Campistol JM, Agusti C, Torras A, Campo E, Abad C, Revert L: Renal hemangioma and renal artery aneurysm in the Klippel-Trenaunay syndrome. J Urol. 1988; 140: 134-6.

7. Fligelstone LJ, Campbell F, Ray DK, Rees RW: The Klippel-Trenaunay syndrome: a rare cause of hematuria requiring nephrectomy. J Urol. 1994; 151: 4045.

8. Yildizdas D, Antmen B, Bayram I, Yapicioglu H: Klippel-trenaunay-Weber syndrome with hydronephrosis and vesicoureteral reflux: an unusual association. Turk J Pediatr. 2002; 44: 180-2.

9. Katsaros D, Grundfest-Broniatowski S: Successful management of visceral Klippel-Trenaunay-Weber syndrome with the antifibrinolytic agent tranexamic acid (cyclocapron): a case report. Am Surg. 1998; 64: 302-4.

10. Klein TW, Kaplan GW: Klippel-Trenaunay syndrome associated with urinary tract hemangiomas. J Urol. 1975; 114: 596-600.

11. Borrelli M, Glina S, Wroclavski ER, Lucon AM, Denes FT, Goes GM: Vesical haemangioma. Report of two cases. Int Urol Nephrol. 1984; 16: 109-14.

12. Kato M, Chiba Y, Sakai K, Orikasa S: Endoscopic neodymium:yttrium aluminium garnet (Nd:YAG) laser irradiation of a bladder hemangioma associated with Klippel-Weber syndrome. Int J Urol. 2000; 7: 1458.

13. Vicente J, Salvador J: Neodymium:YAG laser treatment of bladder hemangiomas. Urology. 1990; 36: 305-8.

14. Smith JA Jr, Dixon JA: Neodymium:YAG laser irradiation of bladder hemangioma. Urology. 1984; 24: 134-6. 
15. Shekarriz B, Upadhyay J, Smith C, Kazmers A, Frontera R: Massive hematuria in adults with KlippelTrenaunay syndrome associated with vascular malformation of the bladder. Urol Int. 2000; 64: 226-8.

16. Zini L, Amara N, Graziana JP, Villers A, Biserte J, Mazeman E: Klippel-Trenaunay syndrome and multiple vesical hemangiomas: treatment with Neodymium:YAG laser. Prog Urol. 2001; 11: 1282-4.

17. Lauvetz RW, Malek RS, Husmann DA: Treatment of extensive urethral hemangioma with KTP/532 laser. Lasers Surg Med. 1996; 18: 92-5.

\section{Correspondence address:}

Dr. Francisco Tibor Dénes

Av. Enéas de Carvalho Aguiar, 255 / sala 710F

São Paulo, SP, 05403-000, Brazil

Fax: + $55113069-8081$

E-mail: f.c.denes@br2001.com.br

\section{EDITORIAL COMMENT}

The Klippel-Trenaunay syndrome (KTS) is an unusual congenital anomaly. It causes symptoms due to genitourinary system involvement in approximately $6 \%$ of the cases (1).

The most feared urological complication is recurrent gross hematuria due to bladder involvement, which could be quite difficult to manage. The paper has a substantial number of KTS patients with genitourinary involvement but in this series none of the patients suffered from recurrent bleeding following treatment, which may not always be the case. The authors described 2 cases of gross hematuria, one case was initially treated with radiotherapy, which caused contracted bladder; it is not the recommended treatment in such patients. In the second case a massive hemangiomatous lesion was
18. Maeda K: A successful case of urethral reconstruction in a child with persistent urethral bleeding due to an extensive cavernous haemangioma. Br J Plast Surg. 1984; 37: 536-8.

19. Rebarber A, Roman AS, Roshan D, Blei F: Obstetric management of Klippel-Trenaunay syndrome. Obstet Gynecol. 2004; 104: 1205-8.

20. Jacob AG, Driscoll DJ, Shaughnessy WJ, Stanson AW, Clay RP, Gloviczki P: Klippel-Trenaunay syndrome: spectrum and management. Mayo Clin Proc. 1998; 73: 28-36.

Accepted after revision:

June 10, 2006

successfully treated with Nd: YAG laser in a single session. Another case, which had extensive bladder hemangiomatous lesions, did not cause any bleeding but caused obstruction to the upper tracts. It was managed by insertion of double $\mathbf{J}$ stent through the lesion, which could be technically demanding and lead to local irritation, and precipitation of hematuria. The rest of the cases were dealt straightforwardly.

Most publications regarding genitourinary manifestations in Klippel Trenaunay syndrome comprise of individual case reports and as the presentation is variable there is no gold standard in treatment. Amongst many options available, Nd: YAG laser has been most commonly and successfully employed as the first line treatment for bleeding from these hemangiomatous lesions. Recently a newer 
treatment with systemic alpha-interferon has been used in a single patient with problematic recurrent hematuria with good short-term results (2).

These patients need long term and close follow up. Any benefit of treating bladder and urethral lesions prophylactically remains to be established. However, the lesions in Klippel Trenaunay syndrome are not true hemangiomas but it may be worthwhile to evaluate any long-term role of endothelial cell growth inhibitors like interferon alpha-2a in preventing bleeding.

\section{REFERENCES}

1. Furness PD 3rd, Barqawi AZ, Bisignani G, Decter RM: Klippel Trenaunay Syndrome: 2 case reports and a review of genitourinary manifestations. J Urol. 2001; 166: 1418-20.

2. Ahmed I, Aslam M, Mahfooz A, Hanash K: Genitourinary manifestations of Klippel- Trenaunay syndrome: report of 2 cases managed with systemic interferon. Scand J Urol Nephrol. 2005; 39: 523-6.

Dr. I. Ahmed

Department of Urology

King Faisal Specialist Hosp \& Research Ctr Riyadh, Saudi Arabia

E-mail: ahmedirfan2000@hotmail.com 